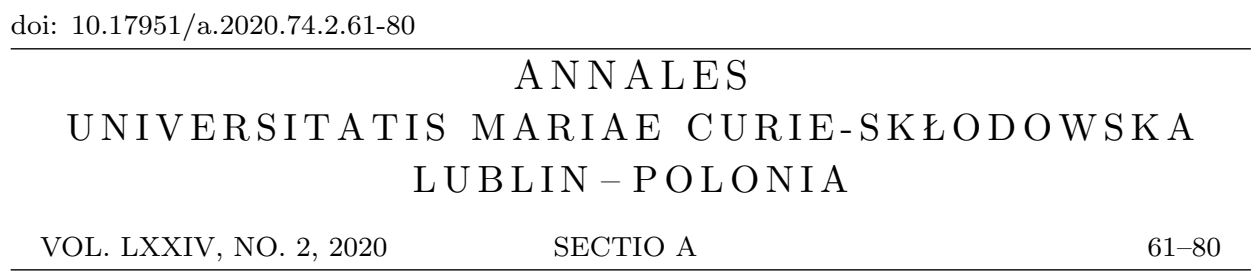

S. H. SAKER, K. LOGAARASI and V. SADHASIVAM

\title{
Forced oscillation of conformable fractional partial delay differential equations with impulses
}

\begin{abstract}
In this paper, we establish some interval oscillation criteria for impulsive conformable fractional partial delay differential equations with a forced term. The main results will be obtained by employing Riccati technique. Our results extend and improve some results reported in the literature for the classical differential equations without impulses. An example is provided to illustrate the relevance of the new theorems.
\end{abstract}

1. Introduction. In recent years, many researchers found that fractional differential equations are more accurate in describing the mathematical modeling of systems and processes in the field of chemical processes, electrodynamics of computer medium, polymer rheology, mathematical biology, etc. The applications of fractional calculus to biomedical problems are done in the areas of membrane biophysics and polymer viscoelasticity, where the experimentally observed power law dynamics for current-voltage and stress-strain relationships are concisely captured by fractional order differential equations. But the most frequently used definitions involve integration which is nonlocal: Riemann-Liouville derivative \& Caputo derivative $[5,9,13,22,27]$. Fractional calculus is the study of derivatives and integrals of non-integer order and is the generalized form of classical derivatives and integrals. Those fractional derivatives in the fractional calculus have seemed complicated and lacked some basic properties, like the product rule and the

2010 Mathematics Subject Classification. 34C10, 34A08, 34A37.

Key words and phrases. Oscillation, fractional differential equations, impulse. 
chain rule. But in 2014, Khalil et al. [12] introduced a new fractional derivative called the conformable derivative which closely resembles the classical derivative.

In order to describe dynamics of populations subject to abrupt changes as well as other phenomena such as harvesting, diseases, and so forth, some authors have used impulsive differential systems to describe the models. For the basic theory on impulsive differential equations, the reader can refer to the monographs and references $[2,16,17,20]$. The study of the qualitative behavior of partial differential equations has rapidly expanded in the last few decades, see for example $[11,14,15,24,25,26,29,30,32]$ and the references they are cited. In particular, the problem of interval oscillation criteria for integer and fractional order impulsive differential equations have been investigated by few authors, we refer the reader to $[3,4,19,28,31]$ and the references cited therein.

Recently, the theory of fractional differential equations has been intensively studied by many authors. For example, we mention to the problem of anomalous diffusion $[7,8]$, the nonlinear oscillation of earthquake which can be modeled with fractional derivative [6], and fluid-dynamic traffic model with fractional derivatives [10] also can be used to eliminate the deficiency arising from the assumption to continuum traffic flow and many other, see also $[18,23]$ and the references they are cited for recent developments in the description of anomalous transport by fractional dynamics. Following this trend, our aim in this paper is to study oscillation properties of partial differential equation of fractional order of the form

$$
\begin{aligned}
& \frac{\partial^{\alpha}}{\partial t^{\alpha}}\left[r(t) g\left(\frac{\partial^{\alpha}}{\partial t^{\alpha}} u(x, t)\right)\right]+\sum_{i=1}^{n} q_{i}(x, t) f_{i}(u(x, t-\sigma)) \\
& =a(t) \Delta u(x, t)+\sum_{j=1}^{m} a_{j}(t) \Delta u\left(x, t-\rho_{j}\right)+F(x, t), \\
& t \neq t_{k}, t \geq t_{0}, \\
& u\left(x, t_{k}^{+}\right)=\alpha_{k}\left(x, t_{k}, u\left(x, t_{k}\right)\right), \\
& \frac{\partial^{\alpha}}{\partial t^{\alpha}} u\left(x, t_{k}^{+}\right)=\beta_{k}\left(x, t_{k}, \frac{\partial^{\alpha}}{\partial t^{\alpha}} u\left(x, t_{k}\right)\right), k=1,2, \ldots, \\
& (x, t) \in \Omega \times \mathbb{R}_{+} \equiv G,
\end{aligned}
$$

where $\Omega$ is a bounded domain in $\mathbb{R}^{N}$ with a piecewise smooth boundary $\partial \Omega$, $\Delta$ is the Laplacian in the Euclidean space $\mathbb{R}^{N}$ and $\mathbb{R}_{+}=[0,+\infty)$, and $\frac{\partial^{\alpha}}{\partial t^{\alpha}}$ denotes the conformable partial fractional derivative of order $\alpha, 0<\alpha \leq 1$. Equation (1.1) is the enhancement with the boundary condition

$$
\frac{\partial u(x, t)}{\partial \gamma}+\mu(x, t) u(x, t)=0 \quad \text { for } \quad(x, t) \in \partial \Omega \times \mathbb{R}_{+},
$$


where $\gamma$ is the outer surface normal vector to $\partial \Omega$ and $\mu(x, t) \in C(\partial \Omega \times$ $[0,+\infty),[0,+\infty))$.

In this paper, we assume that the following hypotheses are satisfied:

$\left(H_{1}\right) r(t) \in C^{\alpha}\left(\mathbb{R}_{+},(0,+\infty)\right), q_{i}(x, t) \in C\left(\bar{G}, \mathbb{R}_{+}\right), q_{i}(t)=\min _{x \in \bar{\Omega}} q_{i}(x, t)$, $i=1,2, \ldots, n, f_{i} \in C(\mathbb{R}, \mathbb{R})$ are convex in $\mathbb{R}_{+}$with $u f(u)>0$, $u f_{i}(u)>0$ and $\frac{f(u)}{u} \geq \epsilon>0, \frac{f_{i}(u)}{u} \geq k_{i}>0$ for $u \neq 0, i=1,2, \ldots, n$, $t-\sigma<t, t-\rho_{j}<t$, for $j=1,2, \ldots, m$.

$\left(H_{2}\right) F \in C(\bar{G}, \mathbb{R}), g \in C(\mathbb{R}, \mathbb{R})$ is convex in $\mathbb{R}_{+}$with $u g(u)>0, g(u) \leq \theta u$ for $u \neq 0, g^{-1} \in C(\mathbb{R}, \mathbb{R})$ is continuous function with $u g^{-1}(u)>0$ for $u \neq 0$ and there exists a positive constant $\eta$ such that $g^{-1}(u v) \leq$ $\eta g^{-1}(u) g^{-1}(v)$ for $u v \neq 0$.

$\left(H_{3}\right) a(t), a_{j}(t) \in P C\left(\mathbb{R}_{+}, \mathbb{R}_{+}\right), j=1,2, \ldots, m$, where $P C$ represents the class of functions which are piecewise continuous in $t$ with discontinuities of first kind only at $t=t_{k}, k=1,2, \ldots$, and left continuous at $t=t_{k}, k=1,2, \ldots$.

$\left(H_{4}\right) u(x, t)$ and its derivative $\frac{\partial^{\alpha}}{\partial t^{\alpha}} u(x, t)$ are piecewise continuous in $t$ with discontinuities of first kind only at $t=t_{k}, k=1,2, \ldots$, and left continuous at $t=t_{k}, u\left(x, t_{k}\right)=u\left(x, t_{k}^{-}\right), \frac{\partial^{\alpha}}{\partial t^{\alpha}} u\left(x, t_{k}\right)=\frac{\partial^{\alpha}}{\partial t^{\alpha}} u\left(x, t_{k}^{-}\right)$ and $0<t_{1}<\cdots<t_{k}<\ldots, \lim _{t \rightarrow+\infty} t_{k}=+\infty$.

$\left(H_{5}\right) \alpha_{k}, \beta_{k} \in P C\left(\bar{\Omega} \times \mathbb{R}_{+} \times \mathbb{R}, \mathbb{R}\right), k=1,2, \ldots$, and there exist positive constants $a_{k}, a_{k}^{*}, b_{k}, b_{k}^{*}$ such that $a_{k}^{*} \leq a_{k} \leq b_{k}^{*} \leq b_{k}$ for $k=1,2, \ldots$ and

$$
a_{k}^{*} \leq \frac{\alpha_{k}\left(x, t_{k}, u\left(x, t_{k}\right)\right)}{u\left(x, t_{k}\right)} \leq a_{k}, \quad b_{k}^{*} \leq \frac{\beta_{k}\left(x, t_{k}, \frac{\partial^{\alpha}}{\partial t^{\alpha}} u\left(x, t_{k}\right)\right)}{\frac{\partial^{\alpha}}{\partial t^{\alpha}} u\left(x, t_{k}\right)} \leq b_{k} .
$$

$\left(H_{6}\right)$ For any $T \geq 0$ there exist intervals $\left[c_{1}, d_{1}\right]$ and $\left[c_{2} . d_{2}\right]$ contained in $[T, \infty)$ such that $c_{1}<d_{1} \leq d_{1}+\sigma \leq c_{2}<d_{2}, c_{l}, d_{l} \notin\left\{t_{k}\right\}, l=1,2$, $k=1,2, \ldots, r(t)>0, q(t) \geq 0$, for $t \in\left[c_{1}-\sigma, d_{1}\right] \cup\left[c_{2}-\sigma, d_{2}\right]$ and $F(t)$ has different signs in $\left[c_{1}-\sigma, d_{1}\right]$ and $\left[c_{2}-\sigma, d_{2}\right]$. For instance we assume that $F(t) \leq 0$ for $t \in\left[c_{1}-\sigma, d_{1}\right]$ and $F(t) \geq 0$ for $t \in\left[c_{2}-\sigma, d_{2}\right]$.

For simplicity, we denote

$$
\begin{aligned}
I(s) & :=\max \left\{j: t_{0}<t_{j}<s\right\}, r_{l}:=\max \left\{r(t): t \in\left[c_{l}, d_{l}\right]\right\}, \quad l=1,2, \\
L_{v}\left(c_{l}, d_{l}\right) & =\left\{v \in C^{\alpha}\left[c_{l}, d_{l}\right], \quad v(t) \not \equiv 0, v\left(c_{l}\right)=v\left(d_{l}\right)=0, l=1,2\right\} .
\end{aligned}
$$

For two constants $c, d \neq t_{k}$ with $c<d$ and a function $\psi \in C([c, d], \mathbb{R})$, we define the operator $\Theta: C([c, d], \mathbb{R}) \rightarrow \mathbb{R}$ by

$$
\Theta_{c}^{d}[\psi]= \begin{cases}0, & I(c)=I(d) \\ \psi\left(t_{I(c)+1}\right) \theta(c)+\sum_{i=I(c)+2}^{I(d)} \psi\left(t_{i}\right) \varepsilon\left(t_{i}\right), & I(c)<I(d),\end{cases}
$$


where

$$
\theta(c)=\frac{a_{I(c)+1}-b_{I(c)+1}}{a_{I(c)+1}\left(t_{I(c)+1}^{\alpha}-c^{\alpha}\right)} \text { and } \varepsilon\left(t_{i}\right)=\frac{a_{i}-b_{i}}{a_{i}\left(t_{i}^{\alpha}-t_{i-1}^{\alpha}\right)} .
$$

The paper is organized as follows: in Section 2, we present some definitions and results that will be needed later. In Section 3, we establish some interval oscillation criteria for the problem (1.1)-(1.2). An example to illustrate our main results is given. To the best of authors' knowledge there has been no work done on the interval oscillation of conformable fractional impulsive partial delay differential equations.

2. Preliminaries. In this section, we present the basic definitions and the basic lemma that will be used in the proof of the main results.

Definition 2.1. A solution $u$ of the problem (1.1)-(1.2) is a function $u \in$ $C^{\alpha}\left(\bar{\Omega} \times\left[t_{-1},+\infty\right), \mathbb{R}\right) \cap C\left(\bar{\Omega} \times\left[\hat{t}_{-1},+\infty\right), \mathbb{R}\right)$ that satisfies (1.1), where

$$
t_{-1}:=\min \left\{0, \min _{1 \leq j \leq m}\left\{\inf _{t \geq 0} t-\rho_{j}\right\}\right\}, \quad \hat{t}_{-1}:=\min \left\{0, \inf _{t \geq 0} t-\sigma\right\} .
$$

Definition 2.2. The solution $u$ of the problem (1.1)-(1.2) is said to be oscillatory in the domain $G$, if it has arbitrary large zeros. Otherwise it is non-oscillatory.

Definition $2.3([12])$. Given $f:[0, \infty) \rightarrow \mathbb{R}$, the conformable fractional derivative of $f$ of order $\alpha$ is defined by

$$
T_{\alpha}(f)(t)=\lim _{\epsilon \rightarrow 0} \frac{f\left(t+\epsilon t^{1-\alpha}\right)-f(t)}{\epsilon}
$$

for all $t>0, \alpha \in(0,1]$.

If $f$ is $\alpha$-differentiable in some interval $(0, a), a>0$ and $\lim _{t \rightarrow 0^{+}} f^{(\alpha)}(t)$ exists, then define

$$
f^{(\alpha)}(0)=\lim _{t \rightarrow 0^{+}} f^{(\alpha)}(t) .
$$

Definition 2.4. $I_{\alpha}^{a}(f)(t)=I_{1}^{a}\left(t^{\alpha-1} f\right)=\int_{a}^{t} \frac{f(x)}{x^{1-\alpha}} d x$, where the integral is the usual Riemann improper integral, and $\alpha \in(0,1)$.

Definition $2.5([1])$. Let $f$ be a function with $n$ variables $x_{1}, x_{2}, \ldots, x_{n}$. The conformable partial derivative of $f$ of order $0<\alpha \leq 1$ in $x_{i}$ is defined as follows

$$
\begin{aligned}
& \frac{\partial^{\alpha}}{\partial x_{i}^{\alpha}} f\left(x_{1}, x_{2}, \ldots, x_{n}\right) \\
& \quad=\lim _{\epsilon \rightarrow 0} \frac{f\left(x_{1}, x_{2}, \ldots, x_{i-1}, x_{i}+\epsilon x_{i}^{1-\alpha}, \ldots, x_{n}\right)-f\left(x_{1}, x_{2}, \ldots, x_{n}\right)}{\epsilon} .
\end{aligned}
$$


Conformable fractional derivatives have the following properties:

Theorem 2.1. Let $\alpha \in(0,1]$ and $f, g$ be $\alpha$-differentiable at some point $t>0$. Then

(i) $T_{\alpha}(a f+b g)=a T_{\alpha}(f)+b T_{\alpha}(g)$ for all $a, b \in \mathbb{R}$.

(ii) $T_{\alpha}\left(t^{p}\right)=p t^{p-\alpha}$ for all $p \in \mathbb{R}$.

(iii) $T_{\alpha}(\lambda)=0$ for all constant functions $f(t)=\lambda$.

(iv) $T_{\alpha}(f g)=f T_{\alpha}(g)+g T_{\alpha}(f)$.

(v) $T_{\alpha}\left(\frac{f}{g}\right)=\frac{g T_{\alpha}(f)-f T_{\alpha}(g)}{g^{2}}$.

(vi) If $f$ is differentiable, then $T_{\alpha}(f)(t)=t^{1-\alpha} \frac{d f}{d t}(t)$.

For convenience, we introduce the following notations:

$$
Y(t)=\frac{1}{|\Omega|} \int_{\Omega} u(x, t) d x, \text { where }|\Omega|=\int_{\Omega} d x,
$$

and

$$
F(t)=\frac{1}{|\Omega|} \int_{\Omega} F(x, t) d x .
$$

The following lemma will be the basic tool in proving the main results.

Lemma 2.1. If the impulsive conformable fractional differential inequality

$$
\left.\begin{array}{l}
T_{\alpha}\left[r(t) g\left(T_{\alpha}(Y(t))\right)\right]+\sum_{i=1}^{n} k_{i} q_{i}(t) Y(t-\sigma) \leq F(t), \quad t \neq t_{k}, \\
a_{k}^{*} \leq \frac{Y\left(t_{k}^{+}\right)}{Y\left(t_{k}\right)} \leq a_{k}, \quad b_{k}^{*} \leq \frac{T_{\alpha}\left(Y\left(t_{k}^{+}\right)\right)}{T_{\alpha}\left(Y\left(t_{k}\right)\right)} \leq b_{k}, \quad k=1,2, \ldots
\end{array}\right\}
$$

has no eventually positive solution, then every solution of the problem (1.1)(1.2) is oscillatory in $G$.

Proof. Assume that there exists a nonoscillatory solution $u(x, t)$ of the problem (1.1)-(1.2) and $u(x, t)>0$. By the assumptions, there exists $t_{1}>$ $t_{0}>0$ such that $t-\sigma \geq t_{1}$ and $t-\rho_{j} \geq t_{1}$ for $t \geq t_{1}>0$, and

$$
\begin{aligned}
& u(x, t-\sigma)>0 \text { for }(x, t-\sigma) \in \Omega \times\left[t_{1}, \infty\right), \\
& u\left(x, t-\rho_{j}\right)>0 \text { for }\left(x, t-\rho_{j}\right) \in \Omega \times\left[t_{1}, \infty\right), j=1,2, \ldots, m .
\end{aligned}
$$

By multiplying both sides of the main equation in (1.1) by $\frac{1}{|\Omega|}$ and integrating with respect to $x$ over the domain $\Omega$, we obtain $t \geq t_{1}$ and $t \neq t_{k}$, 
$k=1,2, \ldots$, such that

$$
\begin{aligned}
& \frac{\partial^{\alpha}}{\partial t^{\alpha}}\left[r(t) g\left(\frac{\partial^{\alpha}}{\partial t^{\alpha}}\left(\frac{1}{|\Omega|} \int_{\Omega} u(x, t) d x\right)\right)\right] \\
& \quad+\frac{1}{|\Omega|} \sum_{i=1}^{n} \int_{\Omega} q_{i}(x, t) f_{i}(u(x, t-\sigma)) d x \\
& =a(t) \frac{1}{|\Omega|} \int_{\Omega} \Delta u(x, t) d x+\frac{1}{|\Omega|} \sum_{j=1}^{m} a_{j}(t) \int_{\Omega} \Delta u\left(x, t-\rho_{j}\right) d x \\
& \quad+\frac{1}{|\Omega|} \int_{\Omega} F(x, t) d x
\end{aligned}
$$

From Green's formula and boundary condition (1.2) we see that

$$
\begin{aligned}
\int_{\Omega} \Delta u(x, t) d x & =\int_{\partial \Omega} \frac{\partial u(x, t)}{\partial \gamma} d S \\
& =-\int_{\partial \Omega} \mu(x, t) u(x, t) d S \leq 0
\end{aligned}
$$

and for $j=1,2, \ldots, m$, we have

$$
\begin{aligned}
\int_{\Omega} \Delta u\left(x, t-\rho_{j}\right) d x & =\int_{\partial \Omega} \frac{\partial u\left(x, t-\rho_{j}\right)}{\partial \gamma} d S \\
& =-\int_{\partial \Omega} \mu(x, t) u\left(x, t-\rho_{j}\right) d S \leq 0,
\end{aligned}
$$

where $d S$ is the surface element on $\partial \Omega$. Moreover by $\left(H_{1}\right)$, it follows that for $i=1,2, \ldots, n$,

$$
\begin{aligned}
\int_{\Omega} q_{i}(x, t) f_{i}(u(x, t-\sigma)) d x & \geq q_{i}(t) \int_{\Omega} f_{i}(u(x, t-\sigma)) d x \\
& \geq k_{i} q_{i}(t) \int_{\Omega} u(x, t-\sigma) d x .
\end{aligned}
$$

In view of $(2.3)-(2.6)$, we obtain

$$
T_{\alpha}\left[r(t) g\left(T_{\alpha}(Y(t))\right)\right]+\sum_{i=1}^{n} k_{i} q_{i}(t) Y(t-\sigma) \leq F(t), \quad t \neq t_{k} .
$$

For $t \geq t_{0}, t=t_{k}, k=1,2, \ldots$, from the boundary conditions we see that

$$
a_{k}^{*} \leq \frac{u\left(x, t_{k}^{+}\right)}{u\left(x, t_{k}\right)} \leq a_{k}, \quad b_{k}^{*} \leq \frac{\frac{\partial^{\alpha}}{\partial t^{\alpha}} u\left(x, t_{k}^{+}\right)}{\frac{\partial^{\alpha}}{\partial t^{\alpha}} u\left(x, t_{k}\right)} \leq b_{k} .
$$

Multiplying both sides by $\frac{1}{|\Omega|}$, integrating with respect to $x$ over the domain $\Omega$, and using $\left(H_{5}\right)$, we obtain

$$
a_{k}^{*} \leq \frac{Y\left(t_{k}^{+}\right)}{Y\left(t_{k}\right)} \leq a_{k}, \quad b_{k}^{*} \leq \frac{T_{\alpha}\left(Y\left(t_{k}^{+}\right)\right)}{T_{\alpha}\left(Y\left(t_{k}\right)\right)} \leq b_{k} .
$$


Hence we see that $Y(t)$ is an eventually positive solution of (2.2). This contradicts our assumption and complete the proof.

3. Main Results. In this section, we establish some new interval oscillation criteria for (1.1) by using the Riccati transformation technique and integral average method. For simplicity, we define

$$
\begin{aligned}
\Gamma_{0}\left(c_{l}, d_{l}\right):= & \int_{c_{l}}^{t_{I\left(c_{l}\right)+1}}\left[\delta\left(v^{\prime}(t)\right)^{2} t^{2-2 \alpha} r(t)-v^{2}(t) Q(t) M_{I\left(c_{l}\right)}^{l}(t)\right] d t \\
& +\sum_{k=I\left(c_{l}\right)+1}^{I\left(d_{l}\right)-1} \int_{t_{k}}^{t_{k+1}}\left[\delta\left(v^{\prime}(t)\right)^{2} t^{2-2 \alpha} r(t)-v^{2}(t) Q(t) M_{k}^{l}(t)\right] d t \\
& +\int_{t_{I\left(d_{l}\right)}^{d_{l}}}^{d^{2}}\left[\delta\left(v^{\prime}(t)\right)^{2} t^{2-2 \alpha} r(t)-v^{2}(t) Q(t) M_{I\left(d_{l}\right)}^{l}(t)\right] d t \\
& +\int_{c_{l}}^{d_{l}}(1-\alpha) t^{-\alpha} v^{2}(t) w(t) d t,
\end{aligned}
$$

where $Q(t)=\sum_{i=1}^{n} k_{i} q_{i}(t)$ and

$$
M_{k}^{l}(t)= \begin{cases}\frac{\sigma \alpha}{\sigma \alpha a_{k}+b_{k}\left(t^{\alpha}-t_{k}^{\alpha}\right)} \frac{(t-\sigma)^{\alpha}-\left(t_{k}-\sigma\right)^{\alpha}}{t_{k}^{\alpha}-\left(t_{k}-\sigma\right)^{\alpha}}, & t \in\left(t_{k}, t_{k}+\sigma\right), \\ \frac{(t-\sigma)^{\alpha}-t_{k}^{\alpha}}{t^{\alpha}-t_{k}^{\alpha}}, & t \in\left[t_{k}+\sigma, t_{k+1}\right) .\end{cases}
$$

Theorem 3.1. Assume that conditions $\left(H_{1}\right)-\left(H_{6}\right)$ hold. Furthermore, assume there exist $c_{l}, d_{l}$ satisfying $T \leq c_{1}<d_{1}, T \leq c_{2}<d_{2}$ for any $T \geq 0$.

(i) $\int_{t_{0}}^{\infty} s^{\alpha-1} g^{-1}\left(\frac{1}{r(s)}\right) d s=\infty$.

(ii) Assume that $v(t) \in L_{v}\left(c_{l}, d_{l}\right)$ is such that

$$
\Gamma_{0}\left(c_{l}, d_{l}\right)<r_{l} \Theta_{c_{l}}^{d_{l}}\left[v^{2}(t)\right]
$$

for $I\left(c_{l}\right)<I\left(d_{l}\right), l=1,2$, then every solution of the problem (1.1)-(1.2) is oscillatory.

Proof. Assume to the contrary that $u(x, t)$ is a non-oscillatory solution of (1.1)-(1.2). Without loss of generality we may assume that $u(x, t)$ is an eventually positive solution of (1.1)-(1.2). Then there exists $t_{1} \geq t_{0}$ such that $u(x, t)>0$ for $t \geq t_{1}$. Therefore, from (2.2) it follows that

$$
T_{\alpha}\left[r(t) g\left(T_{\alpha}(Y(t))\right)\right] \leq F(t)-Q(t) Y(t-\sigma) \text { for } t \in\left[t_{1}, \infty\right) .
$$

Thus $T_{\alpha}(Y(t)) \geq 0$ or $T_{\alpha}(Y(t))<0, t \geq t_{1}$, for some $t_{1} \geq t_{0}$. We now claim that

$$
T_{\alpha}(Y(t)) \geq 0 \text { for } t \geq t_{1} \text {. }
$$


Suppose not, then $T_{\alpha}(Y(t))<0$ and there exists $t_{2} \in\left[t_{1}, \infty\right)$ such that $T_{\alpha}\left(Y\left(t_{2}\right)\right)<0$. Since $r(t) g\left(T_{\alpha}(Y(t))\right)$ is strictly decreasing in $\left[t_{1}, \infty\right)$, it is clear that

$$
r(t) g\left(T_{\alpha}(Y(t))\right)<r\left(t_{2}\right) g\left(T_{\alpha}\left(Y\left(t_{2}\right)\right)\right):=-c,
$$

where $c>0$ is a constant. For $t \in\left[t_{2}, \infty\right)$, after integrating the above inequality from $t_{2}$ to $t$, we have

$$
Y(t) \leq Y\left(t_{2}\right)-c_{1} \int_{t_{2}}^{t} s^{\alpha-1} g^{-1}\left(\frac{1}{r(s)}\right) d s .
$$

Letting $t \rightarrow \infty$, we get $\lim _{t \rightarrow+\infty} Y(t)=-\infty$. This contradiction shows that (3.3) holds. Define the Riccati transformation

$$
w(t):=\frac{r(t) g\left(T_{\alpha}(Y(t))\right)}{Y(t)} .
$$

It follows from (2.2) that $w(t)$ satisfies

$$
T_{\alpha}(w(t)) \leq \frac{F(t)}{Y(t)}-Q(t) \frac{Y(t-\sigma)}{Y(t)}-\frac{w^{2}(t)}{\delta r(t)} .
$$

By assumption $\left(H_{6}\right)$, we can choose $c_{1}, d_{1} \geq t_{0}$ such that $r(t)>0, q(t) \geq 0$ for $t \in\left[c_{1}-\sigma, d_{1}\right]$ and $F(t) \leq 0$ for $t \in\left[c_{1}-\sigma, d_{1}\right]$. From (2.2), we can easily see that

$$
T_{\alpha}(w(t)) \leq-Q(t) \frac{Y(t-\sigma)}{Y(t)}-\frac{w^{2}(t)}{\delta r(t)} .
$$

For $t=t_{k}, k=1,2, \ldots$, we have

$$
w\left(t_{k}^{+}\right):=\frac{r\left(t_{k}^{+}\right) g\left(T_{\alpha}\left(Y\left(t_{k}^{+}\right)\right)\right)}{Y\left(t_{k}^{+}\right)} \leq \frac{b_{k}}{a_{k}} w\left(t_{k}\right) .
$$

First, we consider the case when $I\left(c_{1}\right)<I\left(d_{1}\right)$. In this case, all the impulsive moments in $\left[c_{1}, d_{1}\right]$ are $t_{I\left(c_{1}\right)+1}, t_{I\left(c_{1}\right)+2}, \ldots, t_{I\left(d_{1}\right)}$. Choose $v(t) \in L_{v}\left(c_{1}, d_{1}\right)$. Multiplying both sides of (3.5) by $v^{2}(t)$ and integrating the resulting inequality from $c_{1}$ to $d_{1}$, we obtain

$$
\begin{aligned}
& \int_{c_{1}}^{t_{I\left(c_{1}\right)+1}} v^{2}(t) t^{1-\alpha} w^{\prime}(t) d t+\int_{t_{I\left(c_{1}\right)+1}}^{t_{I\left(c_{1}\right)+2}} v^{2}(t) t^{1-\alpha} w^{\prime}(t) d t+\ldots \\
& \quad+\int_{t_{I\left(d_{1}\right)}}^{d_{1}} v^{2}(t) t^{1-\alpha} w^{\prime}(t) d t \\
& \leq-\int_{c_{1}}^{t_{I\left(c_{1}\right)+1}} v^{2}(t) \frac{w^{2}(t)}{\delta r(t)} d t-\int_{t_{I\left(c_{1}\right)+1}}^{t_{I\left(c_{1}\right)+2}} v^{2}(t) \frac{w^{2}(t)}{\delta r(t)} d t-\ldots \\
& \quad-\int_{t_{I\left(d_{1}\right)}}^{d_{1}} v^{2}(t) \frac{w^{2}(t)}{\delta r(t)} d t-\int_{c_{1}}^{t_{I\left(c_{1}\right)+1}} v^{2}(t) Q(t) \frac{Y(t-\sigma)}{Y(t)} d t
\end{aligned}
$$




$$
\begin{aligned}
& -\int_{t_{I\left(c_{1}\right)+1}}^{t_{I\left(c_{1}\right)+1}+\sigma} v^{2}(t) Q(t) \frac{Y(t-\sigma)}{Y(t)} d t \\
& -\int_{t_{I\left(c_{1}\right)+1}+\sigma}^{t_{I\left(c_{1}\right)+2}} v^{2}(t) Q(t) \frac{Y(t-\sigma)}{Y(t)} d t-\ldots \\
& -\int_{t_{I\left(d_{1}\right)-1}+\sigma}^{t_{I\left(d_{1}\right)}} v^{2}(t) Q(t) \frac{Y(t-\sigma)}{Y(t)} d t-\int_{t_{I\left(d_{1}\right)}}^{d_{1}} v^{2}(t) Q(t) \frac{Y(t-\sigma)}{Y(t)} d t .
\end{aligned}
$$

Using integration by parts on the left-hand side, and noting that $v\left(c_{1}\right)=$ $v\left(d_{1}\right)=0$, we get

$$
\begin{aligned}
& \sum_{k=I\left(c_{1}\right)+1}^{I\left(d_{1}\right)} v^{2}\left(t_{k}\right) t_{k}^{1-\alpha}\left[w\left(t_{k}\right)-w\left(t_{k}^{+}\right)\right] \\
\leq & -\int_{c_{1}}^{d_{1}}\left[\frac{v(t) w(t)}{\sqrt{\delta r(t)}}-v^{\prime}(t) t^{1-\alpha} \sqrt{\delta r(t)}\right]^{2} d t \\
& -\int_{c_{1}}^{t_{I\left(c_{1}\right)+1}} v^{2}(t) Q(t) \frac{Y(t-\sigma)}{Y(t)} d t \\
& -\sum_{k=I\left(c_{1}\right)+1}^{I\left(d_{1}\right)-1}\left[\int_{t_{k}}^{t_{k}+\sigma} v^{2}(t) Q(t) \frac{Y(t-\sigma)}{Y(t)} d t\right. \\
& \left.+\int_{t_{I\left(d_{1}\right)}}^{d_{1}} v^{t_{k+1}} v^{2}(t) Q(t) \frac{Y(t-\sigma)}{Y(t)} d t\right] \\
& +\int_{c_{1}}^{d_{1}}(1-\alpha) t^{-\alpha} p^{2}(t) w(t) d t .
\end{aligned}
$$

We consider several cases to estimate $\frac{Y(t-\sigma)}{Y(t)}$.

Case 1: For $t \in\left(t_{k}, t_{k+1}\right] \subset\left[c_{1}, d_{1}\right]$. If $t \in\left(t_{k}, t_{k+1}\right] \subset\left[c_{1}, d_{1}\right]$, since $t_{k+1}-t_{k}>\sigma$, we consider two subcases:

Case 1.1: If $t \in\left[t_{k}+\sigma, t_{k+1}\right]$, then $t-\sigma \in\left[t_{k}, t_{k+1}-\sigma\right]$ and there are no impulsive moments in $(t-\sigma, t)$. Then, for any $t \in\left[t_{k}+\sigma, t_{k+1}\right]$ we have

$$
Y(t)-Y\left(t_{k}^{+}\right)=T_{\alpha}(Y(\xi))\left(\frac{t^{\alpha}-t_{k}^{\alpha}}{\alpha}\right), \quad \xi \in\left(t_{k}, t\right) .
$$

From this,

$$
Y(t) \geq T_{\alpha}(Y(\xi))\left(\frac{t^{\alpha}-t_{k}^{\alpha}}{\alpha}\right)
$$


We obtain

$$
\frac{T_{\alpha}(Y(t))}{Y(t)}<\frac{\alpha}{t^{\alpha}-t_{k}^{\alpha}} .
$$

Integrating it from $t-\sigma$ to $t$, we have

$$
\frac{Y(t-\sigma)}{Y(t)}>\frac{(t-\sigma)^{\alpha}-t_{k}^{\alpha}}{t^{\alpha}-t_{k}^{\alpha}} .
$$

Case 1.2: If $t \in\left(t_{k}, t_{k}+\sigma\right)$, then $t-\sigma \in\left(t_{k}-\sigma, t_{k}\right)$ and there is an impulsive moment $t_{k}$ in $(t-\sigma, t)$. Similarly to Case 1.1, we obtain

$$
Y(t)-Y\left(t_{k}-\sigma\right)=T_{\alpha}\left(Y\left(\xi_{1}\right)\right)\left(\frac{t^{\alpha}-\left(t_{k}-\sigma\right)^{\alpha}}{\alpha}\right), \quad \xi_{1} \in\left(t_{k}-\sigma, t_{k}\right]
$$

or

$$
\frac{T_{\alpha}(Y(t))}{Y(t)}<\frac{\alpha}{\delta} \frac{1}{t^{\alpha}-\left(t_{k}-\sigma\right)^{\alpha}} .
$$

Integrating it from $t-\sigma$ to $t$, we get

$$
\frac{Y(t-\sigma)}{Y\left(t_{k}\right)}>\frac{(t-\sigma)^{\alpha}-\left(t_{k}-\sigma\right)^{\alpha}}{t_{k}^{\alpha}-\left(t_{k}-\sigma\right)^{\alpha}}>0, \quad t \in\left(t_{k}, t_{k}+\sigma\right) .
$$

For any $t \in\left(t_{k}, t_{k}+\sigma\right)$ we have

$$
Y(t)-Y\left(t_{k}^{+}\right) \leq T_{\alpha}\left(Y\left(t_{k}^{+}\right)\right)\left(\frac{t^{\alpha}-t_{k}^{\alpha}}{\alpha}\right) .
$$

Using the impulsive conditions in equation (1.1), we get

$$
\begin{aligned}
Y(t)-a_{k} Y\left(t_{k}\right) & <b_{k} T_{\alpha}\left(Y\left(t_{k}\right)\right)\left(\frac{t^{\alpha}-t_{k}^{\alpha}}{\alpha}\right) \\
\frac{Y(t)}{Y\left(t_{k}\right)} & <b_{k} \frac{T_{\alpha}\left(Y\left(t_{k}\right)\right)}{Y\left(t_{k}\right)}\left(\frac{t^{\alpha}-t_{k}^{\alpha}}{\alpha}\right)+a_{k} .
\end{aligned}
$$

Using $\frac{T_{\alpha}\left(Y\left(t_{k}\right)\right)}{Y\left(t_{k}\right)}<\frac{1}{\sigma}$, we obtain

$$
\frac{Y(t)}{Y\left(t_{k}\right)}<a_{k}+\frac{b_{k}}{\sigma}\left(\frac{t^{\alpha}-t_{k}^{\alpha}}{\alpha}\right) .
$$

That is,

$$
\frac{Y\left(t_{k}\right)}{Y(t)}>\frac{\sigma \alpha}{\sigma \alpha a_{k}+b_{k}\left(t^{\alpha}-t_{k}^{\alpha}\right)} .
$$

From (3.8) and (3.9), we get

$$
\frac{Y(t-\sigma)}{Y(t)}>\frac{\sigma \alpha}{\sigma \alpha a_{k}+b_{k}\left(t^{\alpha}-t_{k}^{\alpha}\right)} \frac{(t-\sigma)^{\alpha}-\left(t_{k}-\sigma\right)^{\alpha}}{t_{k}^{\alpha}-\left(t_{k}-\sigma\right)^{\alpha}} \geq 0 .
$$

Case 2: If $t \in\left[c_{1}, t_{I\left(c_{1}\right)+1}\right]$, we consider three subcases:

Case 2.1: If $t_{I\left(c_{1}\right)}>c_{1}-\sigma$ and $t \in\left[t_{I\left(c_{1}\right)}+\sigma, t_{I\left(c_{1}\right)+1}\right]$, then $t-\sigma \in$ 
$\left[t_{I\left(c_{1}\right)}, t_{I\left(c_{1}\right)+1}-\sigma\right]$ and there are no impulsive moments in $(t-\sigma, t)$. Proceeding as in Case 1.1 and using the Mean-value Theorem on $\left(t_{I\left(c_{1}\right)}, t_{I\left(c_{1}\right)+1}\right]$, we get

$$
\frac{Y(t-\sigma)}{Y(t)}>\frac{(t-\sigma)^{\alpha}-t_{I\left(c_{1}\right)}^{\alpha}}{t^{\alpha}-t_{I\left(c_{1}\right)}^{\alpha}}>0, \quad t \in\left[t_{I\left(c_{1}\right)}+\sigma, t_{I\left(c_{1}\right)+1}\right] .
$$

Case 2.2: If $t_{I\left(c_{1}\right)}>c_{1}-\sigma$ and $t \in\left[c_{1}, t_{I\left(c_{1}\right)}+\sigma\right)$, then $t-\sigma \in\left[c_{1}-\sigma, t_{I\left(c_{1}\right)}\right)$ and there is an impulsive moment $t_{I\left(c_{1}\right)}$ in $(t-\sigma, t)$. Making a similar analysis as in Case 1.2, we have

$$
\frac{Y(t-\sigma)}{Y(t)}>\frac{\sigma \alpha}{\sigma \alpha a_{I\left(c_{1}\right)}+b_{I\left(c_{1}\right)}\left(t^{\alpha}-t_{I\left(c_{1}\right)}^{\alpha}\right)} \frac{(t-\sigma)^{\alpha}-\left(t_{I\left(c_{1}\right)}-\sigma\right)^{\alpha}}{t_{I\left(c_{1}\right)}^{\alpha}-\left(t_{I\left(c_{1}\right)}-\sigma\right)^{\alpha}} \geq 0,
$$

$t \in\left(c_{1}, t_{I\left(c_{1}\right)}+\sigma\right)$.

Case 2.3: If $t_{I\left(c_{1}\right)}<c_{1}-\sigma$, then for any $t \in\left[c_{1}, t_{I\left(c_{1}\right)+1}\right], t-\sigma \in\left[c_{1}-\right.$ $\left.\sigma, t_{I\left(c_{1}\right)+1}-\sigma\right]$ and there are no impulsive moments in $(t-\sigma, t)$. Working as in Case 1.1, we get

$$
\frac{Y(t-\sigma)}{Y(t)}>\frac{(t-\sigma)^{\alpha}-t_{I\left(c_{1}\right)}^{\alpha}}{t^{\alpha}-t_{I\left(c_{1}\right)}^{\alpha}}>0, \quad t \in\left[c_{1}, t_{I\left(c_{1}\right)+1}\right] .
$$

Case 3: For $t \in\left(t_{I\left(d_{1}\right)}, d_{1}\right]$ we consider three subcases:

Case 3.1: If $t_{I\left(d_{1}\right)}+\sigma<d_{1}$ and $t \in\left[t_{I\left(d_{1}\right)}+\sigma, d_{1}\right]$, then $t-\sigma \in\left[t_{I\left(d_{1}\right)}, d_{1}-\sigma\right]$ and there are no impulsive moments in $(t-\sigma, t)$. Using a similar analysis as in Case 2.1, we have

$$
\frac{Y(t-\sigma)}{Y(t)}>\frac{(t-\sigma)^{\alpha}-t_{I\left(d_{1}\right)}^{\alpha}}{t^{\alpha}-t_{I\left(d_{1}\right)}^{\alpha}}>0, \quad t \in\left[t_{I\left(d_{1}\right)}+\sigma, d_{1}\right] .
$$

Case 3.2: If $t_{I\left(d_{1}\right)}+\sigma<d_{1}$ and $t \in\left[t_{I\left(d_{1}\right)}, t_{I\left(d_{1}\right)}+\sigma\right)$, then $t-\sigma \in$ $\left[t_{I\left(d_{1}\right)}-\sigma, t_{I\left(d_{1}\right)}\right)$ and there is an impulsive moment $t_{I\left(d_{1}\right)}$ in $(t-\sigma, t)$. Using a similar analysis as in Case 2.2, we obtain

$$
\frac{Y(t-\sigma)}{Y(t)}>\frac{\sigma \alpha}{\sigma \alpha a_{I\left(d_{1}\right)}+b_{I\left(d_{1}\right)}\left(t^{\alpha}-t_{I\left(d_{1}\right)}^{\alpha}\right)} \frac{(t-\sigma)^{\alpha}-\left(t_{I\left(d_{1}\right)}-\sigma\right)^{\alpha}}{t_{I\left(d_{1}\right)}^{\alpha}-\left(t_{I\left(d_{1}\right)}-\sigma\right)^{\alpha}} \geq 0 .
$$

Case 3.3: If $t_{I\left(d_{1}\right)}+\sigma \geq d_{1}$, then for any $t \in\left(t_{I\left(d_{1}\right)}, d_{1}\right]$ we get $t-\sigma \in$ $\left(t_{I\left(d_{1}\right)}-\sigma, d_{1}-\sigma\right]$ and there is an impulsive moment $t_{I\left(d_{1}\right)}$ in $(t-\sigma, t)$.

Proceeding as in Case 3.2, we get

$$
\frac{Y(t-\sigma)}{Y(t)}>\frac{\sigma \alpha}{\sigma \alpha a_{I\left(d_{1}\right)}+b_{I\left(d_{1}\right)}\left(t^{\alpha}-t_{I\left(d_{1}\right)}^{\alpha}\right)} \frac{(t-\sigma)^{\alpha}-\left(t_{I\left(d_{1}\right)}-\sigma\right)^{\alpha}}{t_{I\left(d_{1}\right)}^{\alpha}-\left(t_{I\left(d_{1}\right)}-\sigma\right)^{\alpha}} \geq 0 .
$$

Combining all these cases, we have

$$
\frac{Y(t-\sigma)}{Y(t)}> \begin{cases}M_{I\left(c_{1}\right)}^{1}(t) & \text { for } t \in\left[c_{1}, t_{I\left(c_{1}\right)+1}\right] \\ M_{k}^{1}(t) & \text { for } t \in\left(t_{k}, t_{k+1}\right], k=I\left(c_{1}\right)+1, \ldots, I\left(d_{1}\right)-1, \\ M_{I\left(d_{1}\right)}^{1}(t) & \text { for } t \in\left(t_{I\left(d_{1}\right)+1}, d_{1}\right] .\end{cases}
$$


Hence, and since $r(t) g\left(T_{\alpha}(Y(t))\right)$ is non-increasing in $\left(c_{1}, t_{I\left(c_{1}\right)+1}\right]$, by (3.7) we have

$$
\begin{aligned}
& \sum_{k=I\left(c_{1}\right)+1}^{I\left(d_{1}\right)} v^{2}\left(t_{k}\right) t_{k}^{1-\alpha}\left[w\left(t_{k}\right)-w\left(t_{k}^{+}\right)\right] \\
& \leq \int_{c_{1}}^{t_{I\left(c_{1}\right)+1}}\left[\left(v^{\prime}(t)\right)^{2} t^{2-2 \alpha} \delta r(t)-v^{2}(t) Q(t) M_{I\left(c_{1}\right)}^{1}(t)\right] d t \\
& \quad+\sum_{k=I\left(c_{1}\right)+1}^{I\left(d_{1}\right)-1} \int_{t_{k}}^{t_{k+1}}\left[\left(v^{\prime}(t)\right)^{2} t^{2-2 \alpha} \delta r(t)-v^{2}(t) Q(t) M_{k}^{1}(t)\right] d t \\
& \quad+\int_{t_{I\left(d_{1}\right)}}^{d_{1}}\left[\left(v^{\prime}(t)\right)^{2} t^{2-2 \alpha} \delta r(t)-v^{2}(t) Q(t) M_{I\left(d_{1}\right)}^{1}(t)\right] d t \\
& \quad+\int_{c_{1}}^{d_{1}}(1-\alpha) t^{-\alpha} v^{2}(t) w(t) d t .
\end{aligned}
$$

Thus

$$
\begin{aligned}
Y(t) & >Y(t)-Y\left(c_{1}\right)=T_{\alpha}\left(Y\left(\xi_{2}\right)\right)\left(\frac{t^{\alpha}-c_{1}^{\alpha}}{\alpha}\right) \\
& \geq \frac{r(t)\left(T_{\alpha}(Y(t))\right)}{r\left(\xi_{2}\right)}\left(\frac{t^{\alpha}-c_{1}^{\alpha}}{\alpha}\right), \quad \xi_{2} \in\left(c_{1}, t\right) .
\end{aligned}
$$

Letting $t \rightarrow t_{I\left(c_{1}\right)+1}^{-}$, it follows that

$$
w\left(t_{I\left(c_{1}\right)+1}\right)<\frac{r_{1}}{t_{I\left(c_{1}\right)+1}^{\alpha}-c_{1}^{\alpha}} .
$$

Similarly, we can prove that on $\left(t_{k-1}, t_{k}\right], k=I\left(c_{1}\right)+2, \ldots, I\left(d_{1}\right)$,

$$
w\left(t_{k}\right)<\frac{r_{1}}{t_{k}^{\alpha}-t_{k-1}^{\alpha}} .
$$

Hence, from (3.11) and (3.12), we have

$$
\begin{aligned}
\sum_{k=I\left(c_{1}\right)+1}^{I\left(d_{1}\right)} v^{2}\left(t_{k}\right) t_{k}^{1-\alpha} w\left(t_{k}\right)\left[\frac{a_{k}-b_{k}}{a_{k}}\right] & \\
\geq & r_{1}\left[v^{2}\left(t_{I\left(c_{1}\right)+1}\right) t_{I\left(c_{1}\right)+1}^{1-\alpha} \frac{a_{I\left(c_{1}\right)+1}-b_{I\left(c_{1}\right)+1}}{a_{I\left(c_{1}\right)+1}} \frac{1}{t_{I\left(c_{1}\right)+1}^{\alpha}-c_{1}^{\alpha}}\right. \\
& \left.+\sum_{k=I\left(c_{1}\right)+1}^{I\left(d_{1}\right)} v^{2}\left(t_{k}\right) t_{k}^{1-\alpha} \frac{a_{k}-b_{k}}{a_{k}} \frac{1}{t_{k}^{\alpha}-t_{k-1}^{\alpha}}\right] \geq r_{1} \Theta_{c_{1}}^{d_{1}}\left[v^{2}(t)\right] .
\end{aligned}
$$


Thus we have

$$
\sum_{k=I\left(c_{1}\right)+1}^{I\left(d_{1}\right)} v^{2}\left(t_{k}\right) t_{k}^{1-\alpha} w\left(t_{k}\right)\left[\frac{a_{k}-b_{k}}{a_{k}}\right] \geq r_{1} \Theta_{c_{1}}^{d_{1}}\left[v^{2}(t)\right] .
$$

Therefore, using (3.10), we get

$$
\begin{aligned}
& \int_{c_{1}}^{t_{I\left(c_{1}\right)+1}}\left[\delta\left(v^{\prime}(t)\right)^{2} t^{2-2 \alpha} r(t)-v^{2}(t) Q(t) M_{I\left(c_{1}\right)}^{1}(t)\right] d t \\
& +\sum_{k=I\left(c_{1}\right)+1}^{I\left(d_{1}\right)-1} \int_{t_{k}}^{t_{k+1}}\left[\delta\left(v^{\prime}(t)\right)^{2} t^{2-2 \alpha} r(t)-v^{2}(t) Q(t) M_{k}^{1}(t)\right] d t \\
& +\int_{t_{I\left(d_{1}\right)}}^{d_{1}}\left[\delta\left(v^{\prime}(t)\right)^{2} t^{2-2 \alpha} r(t)-v^{2}(t) Q(t) M_{I\left(d_{1}\right)}^{1}(t)\right] d t \\
& +\int_{c_{1}}^{d_{1}}(1-\alpha) t^{-\alpha} v^{2}(t) w(t) d t>r_{1} \Theta_{c_{1}}^{d_{1}}\left[v^{2}(t)\right],
\end{aligned}
$$

which contradicts (3.1). If $I\left(c_{1}\right)=I\left(d_{1}\right)$ then $r_{1} \Theta_{c_{1}}^{d_{1}}\left[v^{2}(t)\right]=0$ and there are no impulsive moments in $\left[c_{1}, d_{1}\right]$. Similarly to the proof of $(3.10)$, we obtain

$$
\int_{c_{1}}^{d_{1}}\left[\delta\left(v^{\prime}(t)\right)^{2} t^{2-2 \alpha} r(t)-v^{2}(t) Q(t) M_{I\left(c_{1}\right)}^{1}(t)+v^{2}(t)(1-\alpha) t^{-\alpha} w(t)\right] d t>0 .
$$

This again contradicts our assumption. Finally, if $u(x, t)$ is eventually negative, we can consider $\left[c_{2}, d_{2}\right]$ instead of $\left[c_{1}, d_{1}\right]$ and get a contradiction. The proof is complete.

Next, we establish new oscillation criteria for (1.1)-(1.2) by using the integral average method used in [21] for ordinary differential equations. Let $D=\left\{(t, s): t_{0} \leq s \leq t\right\}$, then the functions $H_{1}, H_{2} \in C(D, \mathbb{R})$ are said to belong to the class $\mathcal{H}$ if

$\left(H_{7}\right) H_{1}(t, t)=H_{2}(t, t)=0, H_{1}(t, s)>0, H_{2}(t, s)>0$ for $t>s$ and

$\left(H_{8}\right) H_{1}$ and $H_{2}$ have partial derivatives $\frac{\partial H_{1}}{\partial t}$ and $\frac{\partial H_{2}}{\partial s}$ on $D$ such that

$$
\frac{\partial H_{1}}{\partial t}=h_{1}(t, s) H_{1}(t, s), \quad \frac{\partial H_{2}}{\partial s}=-h_{2}(t, s) H_{2}(t, s)
$$

where $h_{1}, h_{2} \in L_{l o c}(D, \mathbb{R})$.

We put

$$
\begin{aligned}
\Gamma_{1, l}= & \int_{c_{l}}^{t_{I\left(c_{l}\right)+1}} H_{1}\left(t, c_{l}\right) Q(t) M_{I\left(c_{l}\right)}^{l}(t) d t \\
& +\sum_{k=I\left(c_{l}\right)+1}^{I\left(\lambda_{l}\right)-1} \int_{t_{k}}^{t_{k+1}} H_{1}\left(t, c_{l}\right) Q(t) M_{k}^{l}(t) d t+\int_{t_{I\left(\lambda_{l}\right)}}^{\lambda_{l}} H_{1}\left(t, c_{l}\right) Q(t) M_{I\left(d_{l}\right)}^{l}(t) d t
\end{aligned}
$$




$$
+\int_{c_{l}}^{\lambda_{l}} H_{1}\left(t, c_{l}\right)\left[\frac{w(t)}{\delta r(t)}-t^{1-\alpha} h_{1}\left(t, c_{l}\right)-(1-\alpha) t^{-\alpha}\right] w(t) d t
$$

and

$$
\begin{aligned}
\Gamma_{2, l}= & \int_{\lambda_{l}}^{t_{I\left(\lambda_{l}\right)+1}} H_{2}\left(d_{l}, t\right) Q(t) M_{I\left(\lambda_{l}\right)}^{l}(t) d t \\
& +\sum_{k=I\left(\lambda_{l}\right)+1}^{I\left(d_{l}\right)-1} \int_{t_{k}}^{t_{k+1}} H_{2}\left(d_{l}, t\right) Q(t) M_{k}^{l}(t) d t \\
& +\int_{t_{I\left(d_{l}\right)}^{d_{l}}}^{d_{2}} H_{2}\left(d_{l}, t\right) Q(t) M_{I\left(d_{l}\right)}^{l}(t) d t \\
& +\int_{\lambda_{l}}^{d_{l}} H_{2}\left(d_{l}, t\right)\left[\frac{w(t)}{\delta r(t)}+t^{1-\alpha} h_{2}\left(d_{l}, t\right)-(1-\alpha) t^{-\alpha}\right] w(t) d t .
\end{aligned}
$$

Theorem 3.2. Assume that conditions $\left(H_{1}\right)-\left(H_{6}\right)$ hold. Furthermore, assume that there exist $c_{l}, d_{l}$ satisfying with $c_{1}<\lambda_{1}<d_{1} \leq c_{2}<\lambda_{2}<d_{2}$. If there exists $H_{1}, H_{2} \in \mathcal{H}$ such that $\left(H_{7}\right),\left(H_{8}\right)$ hold and

$$
\frac{1}{H_{1}\left(\lambda_{1}, c_{1}\right)} \Gamma_{1,1}+\frac{1}{H_{2}\left(d_{1}, \lambda_{1}\right)} \Gamma_{2,1}>\Lambda\left(H_{1}, H_{2} ; c_{l}, d_{l}\right),
$$

where

$$
\begin{aligned}
& \Lambda\left(H_{1}, H_{2} ; c_{l}, d_{l}\right) \\
& =-\left\{\frac{r_{l}}{H_{1}\left(\lambda_{l}, c_{l}\right)} \Theta_{c_{l}}^{\lambda_{l}}\left[H_{1}\left(\cdot, c_{l}\right)\right]+\frac{r_{l}}{H_{2}\left(d_{l}, \lambda_{l}\right)} \Theta_{\lambda_{l}}^{d_{l}}\left[H_{2}\left(d_{l}, \cdot\right)\right]\right\},
\end{aligned}
$$

then every solution of (1.1)-(1.2) is oscillatory.

Proof. Suppose to the contrary that there is a non-oscillatory solution $u(x, t)$ of the problem (1.1)-(1.2). Notice that whether or not there are impulsive moments in $\left[c_{1}, \lambda_{1}\right]$ and $\left[\lambda_{1}, d_{1}\right]$, we should consider the following cases $I\left(c_{1}\right)<I\left(\lambda_{1}\right)<I\left(d_{1}\right), I\left(c_{1}\right)=I\left(\lambda_{1}\right)<I\left(d_{1}\right), I\left(c_{1}\right)<I\left(\lambda_{1}\right)=I\left(d_{1}\right)$ and $I\left(c_{1}\right)=I\left(\lambda_{1}\right)=I\left(d_{1}\right)$.

Moreover, the impulsive moments of $Y(t-\sigma)$ involve the following two cases: $t_{I\left(\lambda_{l}\right)}+\sigma>\lambda_{l}$ and $t_{I\left(\lambda_{l}\right)}+\sigma \leq \lambda_{l}$. Consider the case $I\left(c_{1}\right)<$ $I\left(\lambda_{1}\right)<I\left(d_{1}\right)$, with $t_{I\left(\lambda_{l}\right)}+\sigma>\lambda_{l}$. For this case, the impulsive moments are $t_{I\left(\lambda_{1}\right)+1}, t_{I\left(\lambda_{1}\right)+2}, \ldots, t_{I\left(d_{1}\right)}$ in $\left[\lambda_{1}, d_{1}\right]$. Multiplying both sides of (3.5) by $H_{1}\left(t, c_{1}\right)$ and integrating from $c_{1}$ to $\lambda_{1}$, we obtain

$$
\begin{aligned}
\int_{c_{1}}^{\lambda_{1}} H_{1}\left(t, c_{1}\right) T_{\alpha}(w(t)) d t & \leq-\int_{c_{1}}^{\lambda_{1}} H_{1}\left(t, c_{1}\right) Q(t) \frac{Y(t-\sigma)}{Y(t)} d t \\
& -\int_{c_{1}}^{\lambda_{1}} H_{1}\left(t, c_{1}\right) \frac{w^{2}(t)}{\delta r(t)} d t
\end{aligned}
$$


Applying integration by parts on the R.H.S of first integral we get,

$$
\begin{aligned}
& \int_{c_{1}}^{\lambda_{1}} H_{1}\left(t, c_{1}\right) Q(t) \frac{Y(t-\sigma)}{Y(t)} d t \\
& +\int_{c_{1}}^{\lambda_{1}}\left(\frac{w(t)}{\delta r(t)}-t^{1-\alpha} h_{1}\left(t, c_{1}\right)-(1-\alpha) t^{-\alpha}\right) w(t) H_{1}\left(t, c_{1}\right) d t \\
\leq & -\sum_{k=I\left(c_{1}\right)+1}^{I\left(\lambda_{1}\right)} H_{1}\left(t_{k}, c_{1}\right) t_{k}^{1-\alpha}\left[w\left(t_{k}\right)-w\left(t_{k}^{+}\right)\right]-H_{1}\left(\lambda_{1}, c_{1}\right) \lambda_{1}^{1-\alpha} w\left(\lambda_{1}\right) .
\end{aligned}
$$

As in the proof of Theorem 3.1, we divide the interval $\left[c_{1}, \lambda_{1}\right]$ into several parts and calculate the estimation of $Y(t-\sigma) / Y(t)$, to obtain

$$
\begin{gathered}
\int_{c_{1}}^{\lambda_{1}} H_{1}\left(t, c_{1}\right) Q(t) \frac{Y(t-\sigma)}{Y(t)} d t \geq \int_{c_{1}}^{t_{I\left(c_{1}\right)+1}} H_{1}\left(t, c_{1}\right) Q(t) M_{I\left(c_{1}\right)}^{1}(t) d t \\
+\sum_{k=I\left(c_{1}\right)+1}^{I\left(\lambda_{1}\right)-1} \int_{t_{k}}^{t_{k+1}} H_{1}\left(t, c_{1}\right) Q(t) M_{k}^{1}(t) d t \\
+\int_{t_{I\left(\lambda_{1}\right)}^{\lambda_{1}}} H_{1}\left(t, c_{1}\right) Q(t) M_{I\left(\lambda_{1}\right)}^{1}(t) d t
\end{gathered}
$$

From (3.16) and (3.17), we obtain

$$
\begin{aligned}
& \int_{c_{1}}^{t_{I\left(c_{1}\right)+1}} H_{1}\left(t, c_{1}\right) Q(t) M_{I\left(c_{1}\right)}^{1}(t) d t \\
& +\sum_{k=I\left(c_{1}\right)+1}^{I\left(\lambda_{1}\right)-1} \int_{t_{k}}^{t_{k+1}} H_{1}\left(t_{k}, c_{1}\right) Q(t) M_{k}^{1}(t) d t \\
& +\int_{t_{I\left(\lambda_{1}\right)}^{\lambda_{1}}} H_{1}\left(t, c_{1}\right) Q(t) M_{I\left(\lambda_{1}\right)}^{1}(t) d t \\
& +\int_{c_{1}}^{\lambda_{1}}\left[\frac{w(t)}{\delta r(t)}-t^{1-\alpha} h_{1}\left(t, c_{1}\right)-(1-\alpha) t^{-\alpha}\right] w(t) H_{1}\left(t, c_{1}\right) d t \\
& \leq-\sum_{k=I\left(c_{1}\right)+1}^{I\left(\lambda_{1}\right)} H_{1}\left(t_{k}, c_{1}\right) t_{k}^{1-\alpha}\left[\frac{a_{k}-b_{k}}{a_{k}}\right] w\left(t_{k}\right)-H_{1}\left(\lambda_{1}, c_{1}\right) \lambda_{1}^{1-\alpha} w\left(\lambda_{1}\right) .
\end{aligned}
$$


On the other hand, multiplying both sides of $(3.5)$ by $H_{2}\left(d_{1}, t\right)$, integrating from $\lambda_{1}$ to $d_{1}$ and following a similar procedure as above, we get

$$
\begin{aligned}
& \int_{\lambda_{1}}^{t_{I\left(\lambda_{1}\right)+1}} H_{2}\left(d_{1}, t\right) Q(t) M_{I\left(\lambda_{1}\right)}^{1}(t) d t \\
& +\sum_{k=I\left(\lambda_{1}\right)+1}^{I\left(d_{1}\right)-1} \int_{t_{k}}^{t_{k+1}} H_{2}\left(d_{1}, t_{k}\right) Q(t) M_{k}^{1}(t) d t \\
& +\int_{t_{I\left(d_{1}\right)}^{d_{1}}}^{d_{2}} H_{2}\left(d_{1}, t\right) Q(t) M_{I\left(d_{1}\right)}^{1}(t) d t \\
& +\int_{\lambda_{1}}^{d_{1}}\left[\frac{w(t)}{\delta r(t)}+t^{1-\alpha} h_{2}\left(d_{1}, t\right)-(1-\alpha) t^{-\alpha}\right] w(t) H_{2}\left(d_{1}, t\right) d t \\
& \leq-\sum_{k=I\left(\lambda_{1}\right)+1}^{I\left(d_{1}\right)} H_{2}\left(d_{1}, t_{k}\right)\left[\frac{a_{k}-b_{k}}{a_{k}}\right] w\left(t_{k}\right)+H_{2}\left(d_{1}, \lambda_{1}\right) \lambda_{1}^{1-\alpha} w\left(\lambda_{1}\right) .
\end{aligned}
$$

Dividing (3.18) and (3.19) by $H_{1}\left(\lambda_{1}, c_{1}\right)$ and $H_{2}\left(d_{1}, \lambda_{1}\right)$, respectively and summing the resulting inequalities, we get

$$
\begin{aligned}
& \frac{1}{H_{1}\left(\lambda_{1}, c_{1}\right)} \Gamma_{1,1}+\frac{1}{H_{2}\left(d_{1}, \lambda_{1}\right)} \Gamma_{2,1} \\
& \leq-\left[\frac{1}{H_{1}\left(\lambda_{1}, c_{1}\right)} \sum_{k=I\left(c_{1}\right)+1}^{I\left(\lambda_{1}\right)} H_{1}\left(t_{k}, c_{1}\right)\left[\frac{a_{k}-b_{k}}{a_{k}}\right] w\left(t_{k}\right)\right. \\
& \left.\quad+\frac{1}{H_{2}\left(d_{1}, \lambda_{1}\right)} \sum_{k=I\left(\lambda_{1}\right)+1}^{I\left(d_{1}\right)} H_{2}\left(d_{1}, t_{k}\right)\left[\frac{a_{k}-b_{k}}{a_{k}}\right] w\left(t_{k}\right)\right] .
\end{aligned}
$$

Using a similar method as in (3.12), we obtain

$$
\left.\begin{array}{c}
-\sum_{k=I\left(c_{1}\right)+1}^{I\left(\lambda_{1}\right)} H_{1}\left(t_{k}, c_{1}\right)\left[\frac{a_{k}-b_{k}}{a_{k}}\right] w\left(t_{k}\right) \leq-r_{1} \Theta_{c_{1}}^{\lambda_{1}}\left[H_{1}\left(\cdot, c_{1}\right)\right] \\
-\sum_{k=I\left(\lambda_{1}\right)+1}^{I\left(d_{1}\right)} H_{2}\left(d_{1}, t_{k}\right)\left[\frac{a_{k}-b_{k}}{a_{k}}\right] w\left(t_{k}\right) \leq-r_{1} \Theta_{\lambda_{1}}^{d_{1}}\left[H_{2}\left(d_{1}, \cdot\right)\right] .
\end{array}\right\}
$$

From (3.20) and (3.21), we obtain

$$
\begin{aligned}
\frac{1}{H_{1}\left(\lambda_{1}, c_{1}\right)} \Gamma_{1,1}+\frac{1}{H_{2}\left(d_{1}, \lambda_{1}\right)} \Gamma_{2,1} & \leq-\left\{r_{1} \Theta_{c_{1}}^{\lambda_{1}}\left[H_{1}\left(\cdot, c_{1}\right)\right]+r_{1} \Theta_{\lambda_{1}}^{d_{1}}\left[H_{2}\left(d_{1}, \cdot\right)\right]\right\} \\
& \leq \Lambda\left(H_{1}, H_{2} ; c_{l}, d_{l}\right),
\end{aligned}
$$


which is a contradiction to condition (3.14). In the case when $u(x, t)<0$, we take the interval $\left[c_{2}, d_{2}\right]$ instead of the interval $\left[c_{1}, d_{1}\right]$ and proceeding as in the proof of this case, we get a contradiction. The proof is complete.

In the following, we present an example to illustrate the results.

Example 1. Consider the following impulsive partial differential equation

$$
\begin{aligned}
& \frac{\partial^{\frac{1}{2}}}{\partial t^{\frac{1}{2}}}\left(\frac{\partial^{\frac{1}{2}}}{\partial t^{\frac{1}{2}}} u(x, t)\right)+\frac{m}{2} u\left(x, t-\frac{\pi}{8}\right)+\frac{3 m}{2} u\left(x, t-\frac{\pi}{8}\right) \\
& \quad=\frac{2 t}{3} \Delta u(x, t)+\frac{5 t}{3} \Delta u(x, t-\pi)+F(x, t), \\
& t \neq 2 k \pi \pm \frac{\pi}{4}, t \geq t_{0}, \\
& u\left(x, t_{k}^{+}\right)=\frac{1}{2} u\left(x, t_{k}\right), \\
& \frac{\partial^{\frac{1}{2}}}{\partial t^{\frac{1}{2}}} u\left(x, t_{k}^{+}\right)=\frac{3}{2} \frac{\partial^{\frac{1}{2}}}{\partial t^{\frac{1}{2}}} u\left(x, t_{k}\right), \quad k=1,2, \ldots,
\end{aligned}
$$

for $(x, t) \in(0, \pi) \times \mathbb{R}_{+}$, with the boundary condition

$$
u_{x}(0, t)+u(0, t)=u_{x}(\pi, t)+u(\pi, t)=0, \quad t \neq 2 k \pi \pm \frac{\pi}{4}, \quad k=1,2, \ldots
$$

Here $\alpha=\frac{1}{2}, r(t)=\frac{1}{2}, q(t)=m / 4, q_{1}(t)=3 m / 4, m>0$ is a positive constant, $f(u)=f_{1}(u)=g(u)=2 u$,

$$
F(x, t)=2 m e^{-x}\left(\sin \left(t-\frac{\pi}{8}\right)+\frac{1}{4} \cos t\right),
$$

$a_{k}=1 / 2, b_{k}=3 / 2$. Let $\sigma=\frac{\pi}{8}, t_{k+1}-t_{k}=\frac{\pi}{2}>\frac{\pi}{8}$. Also, for any $T>0$ we choose $k$ large enough such that $T<c_{1}=4 k \pi-\frac{\pi}{2}<d_{1}=4 k \pi$ and $c_{2}=4 k \pi+\frac{\pi}{8}<d_{2}=4 k \pi+\frac{\pi}{2}, k=1,2, \ldots$ Then there is an impulsive movement $t_{k}=4 k \pi-\frac{\pi}{4}$ in $\left[c_{1}, d_{1}\right]$ and an impulsive moment $t_{k+1}=4 k \pi+\frac{\pi}{4}$ in $\left[c_{2}, d_{2}\right]$. For $\epsilon=\epsilon_{1}=1 / 2$ we have $Q(t)=m / 2$, and we take $v(t)=\sin 4 t$, $t_{I\left(c_{1}\right)}=4 k \pi-\frac{7 \pi}{4}, t_{I\left(d_{1}\right)}=4 k \pi-\frac{\pi}{4}$. Then by simple calculation, the left-hand side of equation (3.1) becomes the following:

$$
\begin{aligned}
& \int_{c_{l}}^{t_{I\left(c_{l}\right)+1}}\left[\delta\left(v^{\prime}(t)\right)^{2} t^{2-2 \alpha} r(t)-v^{2}(t) Q(t) M_{I\left(c_{l}\right)}^{l}(t)\right] d t \\
& \quad+\sum_{k=I\left(c_{l}\right)+1}^{I\left(d_{l}\right)-1} \int_{t_{k}}^{t_{k+1}}\left[\delta\left(v^{\prime}(t)\right)^{2} t^{2-2 \alpha} r(t)-v^{2}(t) Q(t) M_{k}^{l}(t)\right] d t \\
& \quad+\int_{t_{I\left(d_{l}\right)}}^{d_{l}}\left[\delta\left(v^{\prime}(t)\right)^{2} t^{2-2 \alpha} r(t)-v^{2}(t) Q(t) M_{I\left(d_{l}\right)}^{l}(t)\right] d t \\
& \quad+\int_{c_{l}}^{d_{l}}(1-\alpha) t^{-\alpha} v^{2}(t) w(t) d t
\end{aligned}
$$




$$
\begin{aligned}
& \leq \int_{4 k \pi-\frac{\pi}{2}}^{4 k \pi-\frac{\pi}{4}}\left[16 t \cos ^{2} 4 t-\frac{1}{2} m \sin ^{2} 4 t\left(\frac{\left(t-\frac{\pi}{8}\right)^{\frac{1}{2}}-\left(4 k \pi-\frac{7 \pi}{4}\right)^{\frac{1}{2}}}{t^{\frac{1}{2}}-\left(4 k \pi-\frac{7 \pi}{4}\right)^{\frac{1}{2}}}\right)\right] d t \\
& \quad+\int_{4 k \pi-\frac{\pi}{4}}^{4 k \pi-\frac{\pi}{8}}\left[16 t \cos ^{2} 4 t-\frac{1}{2} m \sin ^{2} 4 t\left(\frac{\frac{\pi}{16}\left[t^{\frac{1}{2}}-\left(4 k \pi-\frac{\pi}{4}\right)^{\frac{1}{2}}\right]^{-1}}{\frac{\pi}{32}+\frac{3}{2}}\right)\right. \\
& \left.\quad \times\left(\frac{\left(t-\frac{\pi}{8}\right)^{\frac{1}{2}}-\left(4 k \pi-\frac{3 \pi}{8}\right)^{\frac{1}{2}}}{\left(4 k \pi-\frac{\pi}{4}\right)^{\frac{1}{2}}-\left(4 k \pi-\frac{3 \pi}{8}\right)^{\frac{1}{2}}}\right)\right] d t \\
& +\int_{4 k \pi-\frac{\pi}{8}}^{4 k \pi}\left[16 t \cos ^{2} 4 t-\frac{1}{2} m \sin ^{2} 4 t\left(\frac{\left(t-\frac{\pi}{8}\right)^{\frac{1}{2}}-\left(4 k \pi-\frac{\pi}{4}\right)^{\frac{1}{2}}}{t^{\frac{1}{2}}-\left(4 k \pi-\frac{7 \pi}{4}\right)^{\frac{1}{2}}}\right)\right] d t \\
& \quad+\frac{1}{2} \int_{4 k \pi-\frac{\pi}{2}}^{4 k \pi} t^{-1} \sin ^{2} 4 t \cot t d t \\
& \simeq 148.099-m 0.26613 .
\end{aligned}
$$

Since $I\left(c_{1}\right)=k-1, I\left(d_{1}\right)=k, r_{1}=2$, we have

$$
r_{1} \Theta_{c_{1}}^{d_{1}}\left[v^{2}(t)\right]=2\left[\frac{a_{I(c)+1}-b_{I(c)+1}}{a_{I(c)+1}\left(t_{I\left(c_{1}\right)+1}^{\alpha}-c^{\alpha}\right)} \sin ^{2}\left(4 t_{I\left(c_{1}\right)+1}\right)\right] .
$$

Note that condition (3.1) is satisfied in $\left[c_{1}, d_{1}\right]$ if

$$
148.0775<m 0.26613
$$

and we can choose the constant $m$ very large enough so that (3.22) holds. Therefore, condition (3.1) is satisfied in $\left[c_{1}, d_{1}\right]$. We can work similarly for $t \in\left[c_{2}, d_{2}\right]$. Hence, by Theorem 3.1 , every solution of (3.22) is oscillatory. In fact, $u(x, t)=e^{-x} \sin t$ is one of such solutions.

\section{REFERENCES}

[1] Atangana, A., Baleanu, D., Alsaedi, A., New properties of conformable derivative, Open Math. 13 (2015), 889-898.

[2] Bainov, D. D., Simenov, P.S., Impulsive Differential Equations: Periodic Solutions and Applications, Longman, Harlow, 1993.

[3] Chatzarakis, G. E., Logaarasi, K., Raja, T., Sadhasivam, V., Interval Oscillation Criteria for Impulsive Conformable Partial Differential Equations, Appl. Anal. Discrete Math. 13 (2019), 325-345.

[4] Chatzarakis, G. E., Logaarasi, K., Raja, T., Sadhasivam, V., Interval oscillation criteria for conformable fractional differential equations with impulses, Applied Mathematics E-Notes, 19 (2019), 354-369.

[5] Chen, D. X., Oscillation criteria of fractional differential equations, Adv. Diff. Equ. 2012 (2012), 1-10.

[6] Delbosco, D., Rodino, L., Existence and uniqueness for a nonlinear fractional differential equation, J. Math. Anal. Appl. 204 (2) (1996), 609-625. 
[7] El-Sayed, A. M. A., Fractional-order diffusion-wave equation, Internat. J. Theoret. Phys. 35 (2) (1996), 311-322.

[8] V. Gafiychuk, B. Datsko and V. Meleshko, Mathematical modeling of time fractional reaction-diffusion systems, J. Comput. Appl. Math. 220 (1-2) (2008), 215-225.

[9] Grace, S. R., Agarwal, R. P., Wong, P. J. Y., Zafer, A., On the oscillation of fractional differential equations, Frac. Calc. Appl. Anal. 15 (2012), 222-231.

[10] He, J., Some applications of nonlinear fractional differential equations and their approximations, Bulletin of Science and Technology 15 (1999), 86-90.

[11] Kalaimani, T., Raja, T., Sadhasivam, V., Saker, S.H., Oscillation of impulsive neutral partial differential equations with distributed deviating arguments, Bul. Math. Soc. Sci. Math. Roumanie (N. S.) 61(109) (2018), 51-68.

[12] Khalil, R. R., Horani, M. Al., Yousef, A., Sababheh, M., A new definition of fractional derivative, J. Com. Appl. Math. 264 (2014), 65-70.

[13] Kilbas, A. A., Srivastava, H. M., Trujillo, J. J., Theory and Applications of Fractional Differential Equations, Elsevier, Amsterdam, 2006.

[14] Kubiaczyk, I., Saker, S. H., Oscillation of parabolic delay differential equations with positive and negative coefficients, Comment. Math. 42 (2002), 221-236.

[15] Kubiaczyk, I., Saker, S. H., Oscillation of delay parabolic differential equations with several coefficients, J. Comp. Appl. Math. 147 (2) (2002), 263-275.

[16] Lakshmikantham, V., Bainov, D. D., Simeonov, P. S., Theory of Impulsive Differential Equations, World Scientific Publishers, Singapore, 1989.

[17] Li, Q. L., Cheung, W. S., Interval oscillation criteria for second-order forced delay differential equations under impulsive effects, Electron. J. Qual. Theor. Diff. Eq. 2013 (43) (2013), 1-11.

[18] Metzler, R., Schick, W., Kilian, H. G, Nonnenmacher, T. F., Relaxation in filled polymers: a fractional calculus approach, Journal of Chemical Physics 103 (1995), $7180-7186$.

[19] Muthulakshmi, V., Thandapani, E., Interval criteria for oscillation of second-order impulsive differential equation with mixed nonlinearities, ,Electron. J. Differ. Eq., 2011(40) (2011), 1-14.

[20] Özbekler, A., Zafer, A., Oscillation of solutions of second order mixed nonlinear differential equations under impulsive perturbations, Comput. Math. Appl. 61 (2011), 933-940.

[21] Philos, Ch. G., Oscillation theorems for linear differential equations of second order, Arch. Math. 53 (1989), 482-492.

[22] Podlubny, I., Fractional Differential Equations, Academic Press, San Diego, 1999.

[23] Ryabov, Y. E., Puzenko, A., Damped oscillations in view of the fractional oscillator equation, Physics Review B 66 (2002), 184-201.

[24] Sadhasivam, V., Logaarasi ,K., Raja, T., Interval oscillation criteria for impulsive partial differential equations, Int. J. Math. and Appl. 6(1-B) (2018), 229-242.

[25] Sadhasivam, V., Raja, T., Logaarasi, K., On the interval oscillation of impulsive partial differential equations with damping term, Int. J. Engg. Sci. Math. 6 (2017), 328-340.

[26] Saker, S. H., Oscillation of hyperbolic nonlinear differential equations with deviating arguments, Publ. Math. Debr. 62 (2003), 165-185.

[27] Samko, S. G., Kilbas, A. A., Maritchev, O. I., Fractional Integrals and Derivatives. Theory and Applications, Gordon and Breach Sci. Publ., Amsterdam, 1993.

[28] Thandapani, E., Manju, E., Pinelas, S., Interval oscillation criteria for second order forced impulsive delay differential equations with damping term, Springer Plus 5 (2016), 1-16. 
[29] Vladimirov, V. S., Equations of Mathematical Physics. Translated from the Russian by Eugene Yankovsky, Nauka, Moscow, 1964.

[30] Wu, J. H., Theory and Applications of Partial Functional Differential Equations, Springer, New York, 1996.

[31] Xiaoliang, Z., Zhonghai, G., Wu-Sheng, W., Interval oscillation criteria for superhalf-linear impulsive differential equations with delay, J. Appl. Math. (2012), art. id. 285051, $22 \mathrm{pp}$.

[32] Yoshida, N., Oscillation Theory of Partial Differential Equations, World Scientific, Singapore, 2008.

S. H. Saker

Mathematics Division

Faculty of Advanced Basic Sciences

Galala University, Galala New City

Egypt

Department of Mathematics

Faculty of Science

Mansoura University

Mansoura 35516

Egypt

e-mail: shsaker@gu.edu.eg

\section{Sadhasivam}

PG and Research Department of Mathematics

Thiruvalluvar Government Arts College

Affil. to Periyar University, Salem - 636011

Rasipuram - 637401

Namakkal (Dt)

Tamil Nadu, India

e-mail: ovsadha@gmail.com

Received February 10, 2020

\section{K. Logaarasi}

Department of Mathematics

Vivekanandha College

of Arts And Sciences

for Women (Autonomous)

Tiruchengode - 637205

Namakkal(Dt)

Tamil Nadu, India

e-mail: logajoni@gmail.com 